\title{
Executive Function in Parkinson's Disease: Set-Shifting or Set-Maintenance?*
}

\author{
Marcus Richards ${ }^{1,3}$, Lucien J. Cote ${ }^{1}$, and Yaakov Stern ${ }^{1,2,3}$ \\ Departments of Neurology ${ }^{1}$, Psychiatry ${ }^{2}$, \\ and the Gertrude H. Sergievski Center ${ }^{3}$, \\ College of Physicians and Surgeons, Columbia University
}

\begin{abstract}
In order to distinguish impairment in set-shifting in Parkinson's disease (PD) from inability to inhibit distraction by stimuli that compete for attention, 18 nondemented patients with idiopathic PD and 13 normal controls equated for age and education, were administered the Odd-Man Out (OMO) test and the Stroop Color-Word Test. PD patients were significantly impaired on the OMO test but showed no evidence of interference during the Stroop test. Analysis of error patterns during the OMO test indicated that the requirement to repeatedly switch rules, rather than the requirement to maintain steady responding between rule switches, was responsible for impaired OMO performance. It is concluded that the OMO test is fundamentally a test of set shifting, rather than a test of set maintenance in PD. In addition, analysis of a larger sample of $P D$ patients revealed a significant positive relationship between number and severity of extrapyramidal signs and error production on the OMO, and between the latter and global mental function. These relationships were independent of each other, suggesting that impairment in set-shifting function in PD may arise from pathology of the fronto-striatal system independently of changes in cognitive ability.
\end{abstract}

\section{INTRODUCTION}

Over the past decade, there has been considerable interest in impaired executive function in Parkinson's disease (see reviews by Brown \& Marsden, 1990; Dubois, Pillon, Agid, \& Boller, 1991; Raskin, Borod, \& Tweedy, 1990). Executive function has been defined as the planning and modulating of ongoing activity in the absence of external guidance (Stern, 1987) and is manifested in a diverse range of tasks, including sequencing, tracking, temporal ordering, and verbal fluency. Disruption of these functions is widely thought to arise from basal ganglia disease and to consequent impairment of frontal control via the "complex" caudatofugal pathways.

* This work was supported by Federal grants AG02802, AG07232, the Parkinson's Disease Foundation and by the Center for Alzheimer's Disease Research in New York City. Address all correspondence to Dr. M. Richards, the Gertrude H. Sergievski Center, 630 West 168th Street, New York, NY 10032, USA.

Accepted for publication: April 23, 1992. 
One aspect of executive function that has received much attention is that of set-shifting. Flowers and Robertson (1985) define "set" as... "a state of brain activity which predisposes a subject to respond in one way when several alternatives are available" (p. 517). Set-shifting, then, is the ability to alter or realign this predisposition when either an external change of task or a self-directed initiative requires one of these alternatives to be chosen and executed (cf. Bowen, 1976; Cools, Van den Bercken, Horstink, Van Spaendonck, \& Berger, 1984). Patients with Parkinson's disease (PD) have impaired performance on numerous tasks that require set-shifting, including the Wisconsin Card Sorting Test (Bowen, Kamienny, Burns, \& Yahr, 1975; Brown \& Marsden, 1988a; Canavan et al., 1989; Lees \& Smith, 1983), part B-part A of the Trail Making Test (Hietanen \& Teravainen, 1986, Taylor, Saint-Cyr, \& Lang, 1987) and a series of fluency, sorting and motor sequencing operations where a change in task demand is imposed midway through execution (Cools et al., 1984). Impairment in set-shifting ability has not been observed uniformly with PD patients, however (Brown \& Marsden, 1988a, Lichter et al., 1988).

There is also evidence that PD patients have difficulty in maintaining set. In particular, Flowers and Robertson (1985) developed the Odd-Man Out Test (OMO), where subjects were required to formulate a rule to determine which stimulus from a set did not belong with the others. After maintaining this rule over successive presentations of stimulus groups, subjects were asked to change the selection rule and maintain this new rule over the next run of trials. Subjects were then required to alternate the rule from one run of trials to another. Under these circumstances, PD patients made significantly more errors than age-matched controls and, unlike controls, did not improve their performance across runs. Also, controls made more errors than patients following each rule switch, but they were able to reduce their error rate through practice as the run progressed. In contrast, errors made by patients were likely to occur at any time within a run. Flowers and Robertson argued that ability to maintain set against competing alternatives is fragile in PD patients.

There are two possible caveats. First, patients and controls were not matched for overall intellectual ability, an important consideration when tasks with a heavy cognitive load such as this one, are used and when a selective rather than a global deficit is inferred from poor performance in these tasks. Second, it is possible that the sporadic pattern of errors made by patients was caused by inability to inhibit distraction by stimuli that compete for attention, independently of the requirement to switch set. For example, Sharpe (1990) has shown that ability to perform an item and sequential memory task is differentially affected by distracting stimuli in PD patients. This author also demonstrated that PD patients responded to more distractors than normals on the unattended input during a dichotic listening task (Sharpe, 1992). It is therefore important to compare OMO performance to performance on a task that assesses the ability to refrain from distraction by nontarget information without the requirement to switch targets. 
A widely used neuropsychological test that assesses the effects of perceptual interference (Talland, 1965) is the Stroop Color-Word Test (Stroop, 1935). In this test, colors denoted by words are different from the color of the ink in which they are printed. While this test has been used in a switching mode, where patients were required to alternate attention between color and word (Brown \& Marsden, 1988b, 1991), its standard method of administration requires subjects to call out the color of the ink only and to inhibit the tendency to read the words. An interference index is obtained by adjusting performance under these conditions for baseline performance in the one or both dimensions alone. Whether PD patients show greater Stroop interference than controls may depend critically on similarities or differences in general intellectual function between the groups. Hietanen and Tervainen (1988) found greater interference in patients with earlyonset PD than in age-matched controls when the color-word score was adjusted for color-only performance. However, the patient group was found to be significantly more impaired than controls on several other cognitive tests. These tests were the Block Design and Picture Completion (though not Similarities) subtests of the Wechsler Adult Intelligence Scale (WAIS; Wechsler, 1955) and the Logical Memory, Visual Reproductions and Associate Learning (though not Digit Span) subtests of the Wechsler Memory Scale (Wechsler, 1945). Interestingly, there was no difference in this Stroop interference measure between a late-onset patient group and age-matched controls, who were more evenly matched for performance in these tests, with poorer scores only on Block Design, Logical Memory and Visual Reproductions. Similarly, Cools et al. (1984) matched patient and control groups for both age and intellectual function, also using selected subtests of the WAIS (Block Design, Picture Completion, Similarities and Vocabulary) and Digit Span, and found no difference between PD patients and controls in degree of Stroop interference.

The present study compared the relative contributions of set-switching and ability to refrain from distraction in the absence of a switching requirement by administering the Odd-Man Out Test and the Stroop Color-Word Interference Test to PD patients and controls matched for age, education and performance on a mental status examination. The presence of a significant Stroop interference effect in PD patients, in combination with an increased and sporadic pattern of OMO errors might suggest that poor OMO performance stems in part from a more general impairment in ability to tune out distracting stimuli. Alternatively, the demonstration of impaired OMO performance in the absence of a Stroop effect would offer proof that PD patients have a specific set-shifting impairment that cannot be accounted for by inability to refrain from responding to nontarget stimuli. 


\section{METHOD}

\section{Subjects}

Forty-five patients with idiopathic PD were recruited during routine neurological outpatient evaluation and provided informed consent. Neurological examination was performed by a senior attending neurologist (LC) during this evaluation. The presence and severity of extrapyramidal signs (EPS) were rated according to the Unified Parkinson's Disease Rating Scale (UPDRS; Stern, 1988). Demographic and clinical characteristics of these patients, including mean Hoehn and Yahr score (Hoehn \& Yahr, 1967) and mean Schwab Activities of Daily Living (ADL) rating (Schwab \& England, 1969) are shown in Table 1.

Forty-one of these patients $(89.1 \%)$ were utilizing dopamine replacement therapy (levodopa or levodopa/carbidopa combination), 34 (73.9\%) a MAOb inhibitor, 6 (13\%) a dopaminergic agonist, $8(17.4 \%)$ anticholinergic medication and $10(21.7 \%)$ amantadine. Patients with a history or clinical evidence of stroke or other neurodegenerative disease were excluded. All patients scored less than 2 on the Mentation item of the UPDRS; a score of 2 or more is considered to indicate dementia (Mayeux et al., 1990).

All patients were administered the modified Mini-Mental Status Examination (mMMS; Mayeux, Stern, Rosen, \& Leventhal, 1981; Stern, Sano, Paulson, \& Mayeux, 1987). This is a mental status examination based on the Folstein Mini-Mental Status Examination (MMSE; Folstein, Folstein, \& McHugh, 1975). In addition to the orientation, registration, recall and language sections of the MMSE, the mMMS contains expanded attention and calculation, naming and construction sections and includes the Digit Span subtest of the WAIS and a general knowledge section. Maximum score is 57. Eighteen patients with a mMMS score of at least 49 (considered to be within high-functioning range for this test) were equated for age and education with 13 normal elderly subjects. Demographic and clinical characteristics of this subgroup of patients are shown in Table 2.

Twelve of the control subjects were recruited from the local community and one was the spouse of a PD patient. No controls had any neurological condition at the time of testing and none were taking medications which affect the CNS. Means and SDs for age, education and mMMS score for these controls are also shown in Table 2.

\section{Materials and Procedure}

All subjects were administered a modified version of the Odd-Man Out Test (Flowers \& Robertson, 1985). Test materials consisted of two sets of 10 cards, each displaying symbols (either designs or letters) printed in black ink on a white background. Symbols varied in both size (for the designs, large and small; for the letters, upper and lower case) and form (for the designs, shape; for the letters, letter name). Randomization of the stimuli within and across triads was performed in a similar manner to that used by Flowers and Robertson.

Table 1. Demographic and Clinical Characteristics (Means and Standard Deviations) for all PD Patients $(N=45)$.

\begin{tabular}{lrr}
\hline Age & 67.6 & $(5.1)$ \\
Education (years) & 13.7 & $(2.7)$ \\
mMMS & 51.4 & $(2.3)$ \\
Disease duration (years) & 7.8 & $(5.1)$ \\
Hoehn and Yahr Score & 2.6 & $(0.7)$ \\
Schwab ADL rating (\%) & 67.6 & $(25.3)$ \\
\hline
\end{tabular}


Table 2. Demographic and Clinical Characteristics (Means and Standard Deviations) for PD Patients $(n=18)$ and Equated Normal Controls $(n=13)$.

\begin{tabular}{lrrrr}
\hline & \multicolumn{2}{c}{ PDs } & \multicolumn{2}{c}{ Controls } \\
\hline Age & 67.6 & $(5.1)$ & 69.0 & $(3.5)$ \\
Education (years) & 13.7 & $(2.7)$ & 14.6 & $(2.3)$ \\
mMMS & 51.4 & $(2.3)$ & 52.8 & $(1.6)$ \\
Disease duration (years) & 7.6 & $(5.8)$ & - & \\
Hoehn and Yahr Score & 2.5 & $(0.7)$ & - & \\
Schwab ADL rating (\%) & 71.0 & $(26.0)$ & - & \\
\hline
\end{tabular}

Subjects were shown the first card of the first pack and asked to point to one symbol which did not belong with the other two and to maintain the same selection rule over the remaining nine cards within that pack. Subjects were then shown the first card of the second pack and asked to change the selection rule and to maintain this new rule for the remainder of the second pack. The first pack was then readministered and the subject asked to use the initial selection rule, followed again by the second pack, with the subject instructed to use the second rule. After each trial, subjects were informed whether their choice was correct or not but at no time were either selection rules verbalized by the experimenter. If an incorrect choice was made on any trial, the subject was asked to make another choice. If subjects were unable to make a choice on any trial, they were asked to guess until the correct symbol was arrived at by elimination. Trial-by-trial responses were recorded throughout. Since Flowers and Robertson (1985) reported that PD patients made sporadic errors that were distributed throughout each trial run, total correct responses over the four runs (out of 40 ) and total correct over runs 2 to 4 (out of 30 ) were calculated and used as principal dependent measures, the latter representing performance during the "switch" conditions only.

Subjects were also administered the Stroop Color-Word Test, as standardized by Golden (1978). They were shown three kinds of stimuli; black and white words denoting three colors (red, green and blue), where subjects had to read the words out loud, $x$-symbols printed in either red, green or blue ink, where subjects had to name the colors out loud, and words denoting red, green and blue which were printed in these colors, but where the color of the ink was different from the color denoted by the word (color-words). Subjects were asked to concentrate on the color of the ink and to ignore the color denoted by the words. In each case, the number of items read out loud within 45 seconds was recorded. In addition, an interference score was obtained by adjusting the color-word score for baseline word-only and color-only scores, using the formula suggested by Golden (1978):

$$
\text { interference }=w c-((c \times w) /(c+w))
$$

where $w=$ the number of word-only stimuli, $c=$ the number of color-only stimuli and $w c$ $=$ the number of color-word stimuli. Although Stroop scores were obtained from all patients matched with controls, 10 of the remaining patients did not complete this test. Of these, 7 patients experienced visual problems (mostly difficulty distinguishing blue and green, which was not a handicap during the OMO test). The test was not administered to the remaining 3 because of limited time availability. 


\section{RESULTS}

\section{PD Patients Versus Equated Controls}

Preliminary $t$ tests showed no statistical difference between the two groups on age, education or mMMS score. Mean mMMS score for controls was comparable to those for normal elderly individuals in previous studies (e.g., 52.3 for Mayeux et al., 1981; 51.9 for Mayeux, Stern, Sano, Cote, \& Williams, 1987; 53.2 for Stern, Mayeux \& Rosen, 1984).

Means and standard deviations for the total correct OMO score, the total correct switch score and total correct scores for each individual run, along with the Stroop subscores, are shown in Table 3.

MANOVA was used to test for group differences in the total correct OMO score and the four Stroop variables, with group as the between-groups factor ( 2 levels). In order to improve the accuracy of the group match, age and education were used as covariates. Hotelling's $\mathrm{T}^{2}$ test revealed a significant group effect $\left(T^{2}=0.78, p=.019\right)$. Corresponding univariate $\mathrm{F}$ tests, with $(1,26)$ d.f., revealed a significant difference in the following variables: total correct OMO $(F=8.56$, $p=.007)$, Stroop word score $(F=7.57, p=.011)$ and Stroop color-word score $(F$ $=5.74, p=.024)$. The Stroop color and interference scores were not significant.

This analysis indicates that PD patients made significantly more errors during the OMO test than controls. This significance was maintained when total correct responses during the "switch" trials only (i.e., runs 2-4) was substituted as a measure. In contrast, while PD patients were significantly slower than controls in reading word-only and color-word stimuli during the Stroop test, there was no difference between the groups in the measure of interference between the words and colors. Neither was a group difference found in the more conventional interference measure of color-word score corrected for color-only score.

Table 3. Means and Standard Deviations for Each Test Score. PD Patients $(n=18)$ and Equated Controls $(n=13)$.

\begin{tabular}{lrrrrr}
\hline & \multicolumn{2}{c}{ PDs } & \multicolumn{2}{c}{ Controls } \\
\hline OMO & & & & \\
$\quad$ Total score & 30.1 & $(6.8)$ & 35.8 & $(3.2)$ \\
Trials 2-4 & 21.7 & $(5.6)$ & 26.8 & $(2.8)$ \\
Trial 1 & 8.4 & $(2.8)$ & 9.0 & $(0.7)$ \\
Trial 2 & 6.9 & $(2.8)$ & 8.5 & $(1.7)$ \\
Trial 3 & 7.9 & $(1.6)$ & 8.9 & $(1.2)$ \\
$\quad$ Trial 4 & 6.9 & $(3.1)$ & 9.5 & $(1.4)$ \\
Stroop & 101.7 & $(16.6)$ & & \\
$\quad$ Word & 65.0 & $(11.6)$ & 71.0 & $(13.9)$ \\
Color & 38.8 & $(9.4)$ & 47.1 & $(11.0)$ \\
Word-color & -1.5 & $(8.7)$ & 3.6 & $(8.0)$ \\
Interference & & & & & $(7.4)$ \\
\hline
\end{tabular}


The distribution of errors within the "switch" runs was investigated by summing the total number of errors occurring during trials 1-5 ("early" errors) and those occurring during trials 6-10 ("late" errors) during runs 2-4. For PDs, 56.4\% of "switch" errors were early and $43.6 \%$ were late. For controls, the ratio was $63.4 \%$ early to $36.6 \%$ late. A chi-square test revealed no significant difference between the groups in this ratio. Inspection of these data indicated that both controls and patients were likely to make errors throughout the switch runs.

A chi-square test revealed no difference between the groups in the choice of rule (form or size) during the initial run (i.e., the first block of 10 trials) of the OMO. Groups were therefore combined to assess whether choice of first rule influenced performance during the test. A $t$ test revealed significantly poorer performance during the initial run if the size rule was chosen first $(t=3.22, p=$ $.004)$. There was no such difference with any other individual run, nor with the total score or total switch score.

In order to examine dynamic patterns of performance during the OMO test, the four runs of this test were analyzed as repeated measures, using MANOVA for polynomial trend analysis, with group as the between-groups factor, runs as the within-subject factor (4 levels) and age and education as covariates. As expected, the group effect was significant $(F=7.53, p=.011)$. The runs effect was not significant. However, there was a significant Groups $x$ Runs interaction $\left(T^{2}=0.33, p=.048\right)$. Corresponding univariate $\mathrm{F}$ tests indicated a significant difference between the groups in the linear trend $(F=9.47, p=.005)$, although other possible trends (quadratic and cubic) were not significant. The difference in linear trend is illustrated by Fig. 1., where an overall improvement in the performance of controls across the four runs can be observed, compared to an overall declining trend in performance for the PD patients. However, it can also be observed that performance across runs was considerably more uneven for PDs than for controls. Clearly, all subjects were disrupted by the first switch (run 2), although the disruption was more pronounced in PDs. All subjects then showed some degree of recovery following the second switch (run 3), a phenomenon which is not surprising, since they were returning to their initial rule of choice. However, performance in controls continued to improve (run 4), even after the (harder) second rule was reimposed, suggesting these subjects had mastered the task by this stage. In contrast, performance in patients deteriorated to its lowest level for the entire task, indicating a cumulative impairment following repeated switches.

\section{PD Patients Only}

In order to investigate in detail the performance of $\mathrm{PD}$ patients during the OMO test, the distribution of errors was plotted trial-by-trial for each run for the larger group of 45 patients. The presence of a measurable distribution to the errors was tested using a one-sample Kolmogorov-Smirnov test, with $\alpha$ set at .05, for each run and for the three switch runs combined. Because there were 10 trials per run, a null distribution of $10 \%$ of the total errors per trial was tested. On each occa- 


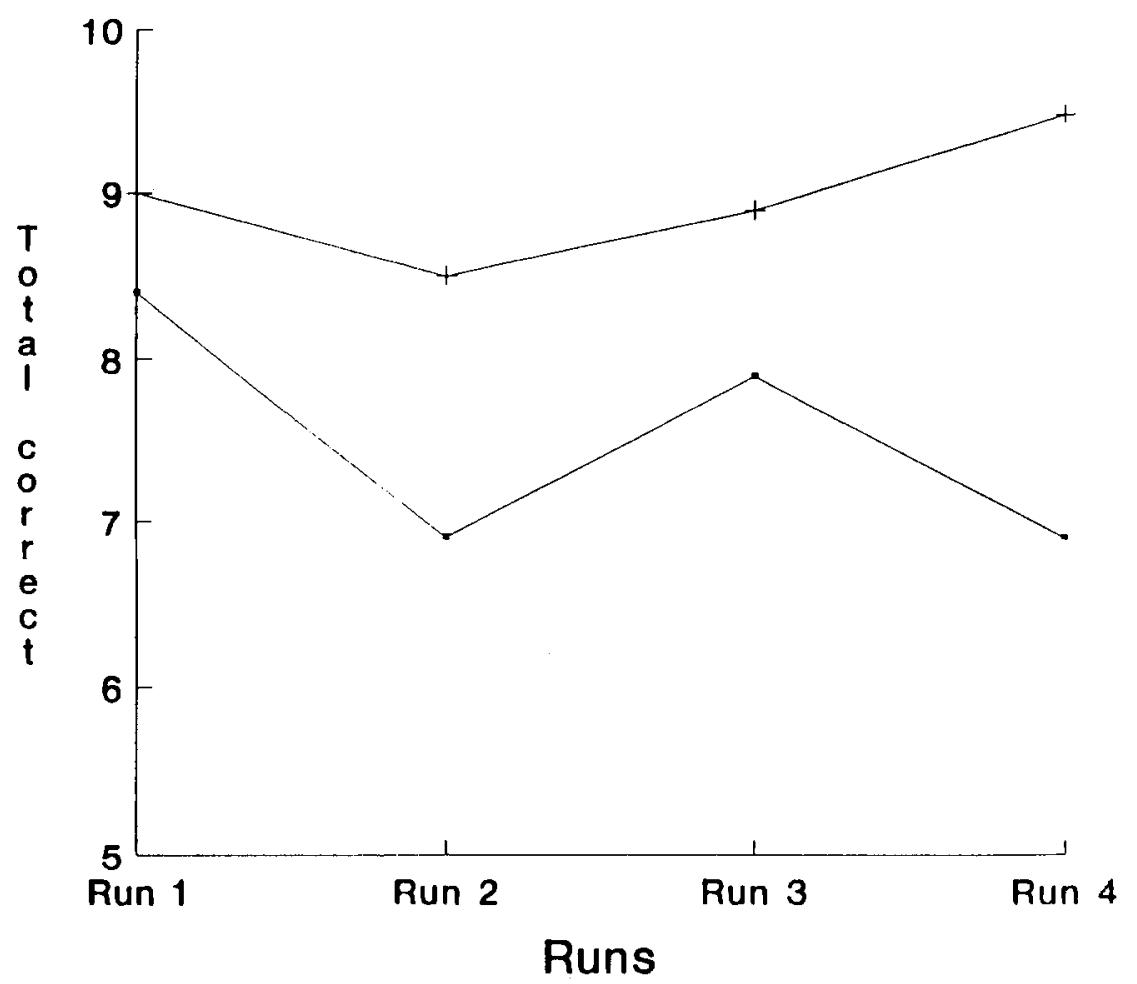

$\rightarrow$ PD patients $\quad-1$ - Controls

Fig. 1. OMO test: run by run scores. PD patients $(n=18)$ and equated controls $(n=13)$.

sion, these tests were unable to reject the null distribution, indicating that errors were likely to occur at any time within each run.

It is generally assumed that impaired executive function in PD arises from disturbances in the fronto-striatal loop. For this reason, the relationship between the neuropsychological test scores and measures of extrapyramidal function are of interest in the present study, especially if this relationship can be established independently of changes in global mental status. The total correct OMO score, the total correct switch OMO score, the total correct scores for the four individual OMO runs and the Stroop subscores were therefore correlated with the total EPS score and the mMMS score. The resulting correlation matrix is shown in Table 4. Intercorrelations between age, mMMS and the EPS score were all nonsignificant and are omitted from the matrix.

Notable are the significant positive correlations between age, the OMO summary scores and the Stroop word-only and color-only scores, and between the 
Table 4. Correlations between Test Scores, Demographic Variables and Indices of PD Severity for all PD Patients $(n=45)$.

\begin{tabular}{lcccccccccc}
\hline & OMO & OMOSW & OMO1 & OMO2 & OMO3 & OMO4 & SW & SC & SCW & SI \\
\hline Age & $-.38^{*}$ & $-.41^{* *}$ & -.13 & $-.34^{*}$ & $-.34^{* *}$ & -.26 & -.32 & $-.39^{*}$ & -.14 & .12 \\
mMMS & $.62^{* * *}$ & $.60^{* * *}$ & $.44^{* *}$ & $.38^{* *}$ & $.60^{* * *}$ & $.35^{*}$ & $.36^{*}$ & $.43^{*}$ & .27 & .03 \\
EPS & $-.38^{*}$ & $-.43^{* *}$ & -.10 & -.22 & $-.36^{*}$ & $-.39^{*}$ & $-.42^{*}$ & -.19 & -.18 & .02
\end{tabular}

$* p<.05 * * p<.01 * * * p<.001$

OMO = total OMO score, OMOSW = OMO switch trials, OMO1-4 = OMO runs 1-4, SW = Stroop word-only score, $\mathrm{SC}=$ Stroop color-only score, $\mathrm{SWC}=$ Stroop color-word score, $\mathrm{SI}=$ Stroop interference score, EPS = total extrapyramidal signs.

mMMS score, the OMO summary scores and the Stroop color-only score. In addition, the EPS score correlated significantly and negatively with the OMO summary measures and, in line with the results of Flowers and Robertson (1985), the negative correlation between the EPS score and OMO performance showed a clear increase in magnitude over the four OMO runs. In contrast, however, there were few correlations between measures of PD severity and the Stroop scores and none between severity and the Stroop interference score. Finally, it should be noted that while both the mMMS score and the total EPS score correlated positively with the OMO summary measures, the two former scores did not correlate with each other.

To clarify these relationships, age, education, total EPS score and the mMMS score were entered into a multiple regression analysis, with the total OMO score as the dependent variable. A forced-entry procedure was used for age and education, and forward-entry was used for the remaining variables. Age and education showed a significant relationship with the total OMO score $(F=4.54, p=.018)$ and accounted for $20.1 \%$ of the variance. Also, the relationships with the addition of the EPS and mMMS scores were significant $(F=7.18, p=.0003$ and $F=7.15$, $p=.0007$, respectively). The mMMS score accounted for an additional $17.9 \%$, and the EPS score accounted for a further $7.8 \%$ of the total OMO variance. However, when these latter two variables were entered, the significance of age and education was lost. Summary regression statistics are presented in Table 5.

Of the four variables entered into the model, therefore, total EPS score and mMMS score were the most significant predictors of OMO performance and both variables independently accounted for variance in OMO performance. When this analysis was repeated for the "switch" trials only, a similar pattern of results emerged. In addition, the amount of variance in this measure accounted for by the EPS score increased to $16.0 \%$ whereas the amount accounted for by the mMMS score decreased to $9.34 \%$, suggesting that performance during this test was more directly influenced by measures of PD severity than by global cognitive function. 
Table 5. Summary Multiple Regression Statistics, with Total OMO Score as Dependent Variable and Age, Education, Total EPS Score and mMMS Score as Independent Variables for all PD Patients $(N=45)$.

\begin{tabular}{lrrr}
\hline Variable & Beta & \multicolumn{1}{c}{$T$} & $p$ \\
\hline Age & 0.109 & 0.781 & n.s. \\
Education & -0.009 & -0.058 & n.s. \\
mMMS & 0.505 & 2.874 & .007 \\
EPS & -0.285 & -2.211 & .034 \\
\hline
\end{tabular}

In contrast, when the multiple regression was repeated on the Stroop test, neither age, education, EPS score or mMMS level showed any significant relationships with either interference score.

\section{DISCUSSION}

PD patients made significantly more errors on the OMO test than age and education-matched controls, overall and when only "switch" runs were taken into consideration. These results therefore replicate the general findings of Flowers and Robertson (1985). Since the two groups were matched for global mental status and since mental status fell within normal range for all subjects, these results represent selective impairment, rather than impairment simply arising from globally compromised cognitive function.

When runs were analyzed as repeated measures, controls showed a drop in accuracy following the first switch run but showed a recovery during the final two runs. An impairment in performance during the second run was also observed with PDs, although unlike the controls, performance for patients remained unstable across runs throughout the remainder of the test. However, unlike the results of Flowers and Robertson, there was no difference between patients and controls in the distribution of errors within the ten trials of each switch run; controls were just as likely to make errors during the later trials of each run as patients. It may be that this distributed error pattern is a natural feature of the OMO test for most subjects. More importantly, these results demonstrate that it is the change of rule from run to run, rather than the requirement to maintain a rule within each run, that causes particular difficulty to $P D$ patients.

In line with the results of Flowers and Robertson (1985), the summary EPS measure correlated positively with the number of OMO errors and the size of this correlation increased across the runs. The mMMS score also correlated with OMO performance, as might be expected since the OMO test has a significant cognitive load. However, multiple regression analyses indicated that extrapyramidal signs and mMMS level independently influenced OMO performance. This diffi- 
culty in set-shifting may be associated with changes in the "complex" caudatofugal pathways that are linked to the prefrontal cortex. Moreover, set-shifting difficulties may parallel impairments in switching motor programs in PD (e.g., Cools et al., 1984; Harrington \& Haaland, 1991), perhaps associated with changes in the motor loop linking the putamen with the premotor fields. In both cases, depletion of dopamine in the striatum is thought to provide a common pathophysiological basis for the changes.

Turning to the Stroop test, there was no evidence from the present study that PD patients were differentially susceptible to Stroop interference. Patients were slower than controls in two conditions, but not in the interference measure, which is corrected for reading speed. In line with this negative finding, measures of PD severity showed no correspondence with those of Stroop interference. The failure of the Stroop test to differentiate PD patients from controls when both groups were equated for global mental status echoes the results of Hietanen and Teravainen (1988) for late-onset patients and of Cools et al. (1984) and can be interpreted in one of two ways.

First, it may be that Stroop performance is particularly vulnerable to normal aging and that indices of Stroop interference are therefore poor discriminators of executive dysfunction in elderly patients. In this context, it is notable that Stern, Tetrud, Martin, Kutner, \& Langston (1990) found significantly greater Stroop interference in patients with MPTP-induced parkinsonism than in controls matched for age and global mental status, when mean age of these subjects was below 35 years.

Second, it may be that the ability to maintain set while exposed to stimuli that compete for attention is preserved in PD. Brown and Marsden (1988b), using a switching version of the Stroop task, found little evidence of difficulty in maintaining set with PD patients. When subjects were required to switch attention between stimulus dimensions (color/word) from run to run, these authors found that performance in PD patients was maximally disrupted immediately following each switch. However, patients were able to overcome this disruption and develop a new set at the same rate as controls as each new trial-run progressed.

In the present experiment, rule switching was shown to differentially affect OMO performance in PD patients when performance across runs (i.e., from rule to rule) was considered. However, both patients and controls showed a similar error distribution within these runs. In both these experiments, changes in response set were shown to be particularly disruptive to PD patients, whereas performance in these patients was no more unstable than controls when periods of response continuity were required (as with the present version of the Stroop). These data offer little support for the notion that ability to maintain set is impaired in $\mathrm{PD}$, but reinforce the more widespread view that these patients are disrupted by requirements to shift set. Furthermore, the demonstration of impairment on OMO performance across sets in PD patients in the absence of a significant difference in Stroop interference militates against the possibility that difficulty with the OMO in $\mathrm{PD}$ patients is underpinned by a general failure to tune out 
distracting information. We suggest that the Odd-Man Out test is fundamentally a test of set-shifting in PD and not a test of set-maintenance or of attention.

In the final analysis, however, the distinction between set-"shifting" and set "maintenance" may be little more than verbal or operational and these terms may not correspond to distinct psychological processes. Set-maintenance can be defined as the preservation of a response (or state of brain activity which predisposes a particular response; cf. Flowers \& Robertson, 1985) in line with a fixed task requirement, or a fixed internal strategy, when one or more response alternatives are possible. On this basis, with the exception of tasks where responding is determined by random or unpredictable contingencies, a requirement to maintain set is present in most tests of executive function, including quintessential "shifting" tasks, such as those requiring response alternation. Set-shifting and setmaintenance, thus conceived, represent opposite (but complimentary) behaviors. The crucial feature of tasks such as the OMO and Brown and Marsden's version of the Stroop is not that they require shifting, but that the shifts themselves are required in the midst of stable responding. With reference to the theory of Shiffrin and Schneider (1977) and the application of this model to cognitive impairment in PD (Weingartner, Burns, Diebel, \& LeWitt, 1984), these circumstances place heavy demand on PD patients by requiring a change from automatic to active control. Alternatively (or in addition), some types of shift may intrinsically place more demands on central processing resources than others (cf. Brown \& Marsden, 1988b). For example, Downes et al. (1989) showed that PD patients could perform reversal tasks as well as normals as long as intra-dimensional shifts were required. However, when a more difficult extra-dimensional shift was introduced, performance in patients was significantly impaired. The simultaneous internal scanning of two sets (letters and numbers) required by part $B$ of the Trail Making test may also be particularly challenging for PD patients. It is the internalization of precisely this kind of behavioral regulation that constitutes the core of executive function (Stern, 1987).

Under some circumstances, however, PD patients do have difficulty in performing tasks in the presence of stimuli that compete for attention, even when no requirement to switch set is made. Sharpe $(1990,1992)$ has shown that performance on memory and dichotic listening tasks is impaired by distracting stimuli to a greater extent in PD patients than in normals. Again, it may be that these tasks are effortful (Weingartner et al., 1984) and/or attention-demanding (Brown \& Marsden, 1988b) and that the addition of distracting stimuli raises task difficulty to a level at which performance becomes significantly impaired in PDs. In this context, Brown and Marsden (1991) have shown that the requirement to perform a concurrent task (random number generation) disrupted performance on their switch version of the Stroop task in PD patients, even when the correct response was cued, an aid that has previously been shown to reduce Stroop interference in patients (Brown \& Marsden, 1988b). It may be that the "primary" task during the standard administration of the Stroop (i.e., reading out the color of the ink) has a relatively low resource requirement so that the presence of distractors (the words) 
does not unduly increase task difficulty in PD patients, even when the distractors are highly competitive for attention. The manner in which distractors exert a disruptive effect on ongoing task performance in PD patients requires further exploration. In particular, it is important to delineate the circumstances under which distractors can operate in their own right and when task demand is increased either by switching between different tasks, or by demanding parallel execution of simultaneous tasks.

\section{REFERENCES}

Bowen, F.P. (1976). Behavioural alterations in patients with basal ganglia lesions. In M.D. Yahr (Ed.), The basal ganglia (pp. 169-180). New York: Raven Press.

Bowen, F.P., Kamienny, R., Burns, M., \& Yahr, M. (1975). Parkinsonism: Effects of levodopa treatment on concept formation. Neurology, 25, 701-704.

Brown, R.G., \& Marsden, C.D. (1988a). An investigation into the phenomenon of 'set' in Parkinson's disease. Movement Disorders, 3, 152-161.

Brown, R.G., \& Marsden, C.D. (1988b). Internal versus external cues and the control of attention in Parkinson's disease. Brain, 111, 323-345.

Brown, R.G., \& Marsden, C.D. (1990). Cognitive function in Parkinson's disease: From description to theory. Trends in Neuroscience, 13, 21-29.

Brown, R.G., \& Marsden, C.D. (1991). Dual task performance and processing resources in normal subjects and patients with Parkinson's disease. Brain, 114, 215-231.

Canavan, A., Passingham, R.E., Marsden, C.D., Quinn, N., Wyke, M., \& Polkey, C.E. (1989). The performance on learning tasks of patients in the early stages of Parkinson's disease. Neuropsychologia, 27, 141-156.

Cools, A.R., Van Den Bercken, J.H.L., Horstink, M.W.I., Van Spaendonck, K.P.M., \& Berger, H.J.C. (1984). Cognitive and motor shifting aptitude disorder in Parkinson's disease. Journal of Neurology, Neurosurgery, and Psychiatry, 47, 443-453.

Downes, J.J., Roberts, A.C., Sahakian, B.J., Evenden, J.L., Morris, R.G., \& Robbins, T.W. (1989). Impaired extra-dimensional shift performance in medicated and unmedicated Parkinson's disease: Evidence for a specific attentional dysfunction. Neuropsychologia, 27, 1329-1343.

Dubois, B., Pillon, B., Agid, Y., \& Boller, F. (1991). Cognitive deficits in Parkinson's disease. In F. Boller \& J. Grafman (Eds.), Handbook of neuropsychology (pp. 195240). New York: Elsevier.

Flowers, K.A., \& Robertson, C. (1985). The effect of Parkinson's disease on the ability to maintain a mental set. Journal of Neurology, Neurosurgery, and Psychiatry, 48, 517 529.

Folstein, M.F., Folstein, S.E., \& McHugh, P. (1975). "Mini-Mental State": A practical method for grading the cognitive state of patients for the clinician. Journal of Psychiatric Research, 12, 189-198.

Golden, C.J. (1978). Stroop color and word test. Chicago: Stoelting Company.

Harrington, D., \& Haaland K. (1991). Sequencing in Parkinson's disease. Abnormalities in programming and controlling movement. Brain, 114, 99-115.

Hietanen, M., \& Teravainen, H. (1986). Cognitive performance in early Parkinson's disease. Acta Neurologica Scandinavica, 73, 151-159.

Hietanen, M., \& Teravainen, H. (1988). The effect of age of disease onset on neuropsychological performance in Parkinson's disease. Journal of Neurology, Neurosurgery, and Psychiatry, 51, 244-249. 
Hoehn, M.M., \& Yahr, M.D. (1967). Parkinson's disease: Onset, progression and mortality. Neurology, 17, 427-442.

Lees, A. \& Smith, E. (1983). Cognitive deficits in the early stages of Parkinson's disease. Brain, 106, 257-270.

Lichter, D.G., Corbett, A.J., Fitzgibbon, G.M., Davidson, O.R., Ayton Hope, J.K., Goddard, G.V., Sharples, K.J., \& Pollock, M. (1988). Cognitive and motor dysfunction in Parkinson's disease. Archives of Neurology, 45, 854-860.

Mayeux, R., Stem, Y., Rosen, J., \& Leventhal, J. (1981). Depression, intellectual impairment, and Parkinson's disease. Neurology, 31, 645-650.

Mayeux, R., Stem, Y., Sano, M., Cote, L., \& Williams, J. (1987). Clinical and biochemical correlates of bradyphrenia in Parkinson's disease. Neurology, 37, 1130-4 (erratum: Neurology, 37, 16931).

Mayeux, R., Chen, J., Mirabello, E., Marder, K., Bell, K., Dooneief, G., Cote, L., \& Stern, Y. (1990). An estimate of the incidence of dementia in idiopathic Parkinson's disease. Neurology, 40, 1513-1517.

Raskin, S., Borod, J., \& Tweedy, J. (1990). Neuropsychological aspects of Parkinson's disease. Neuropsychology Review, 1, 185-221.

Schwab, R.S., \& England, A.C. Jr. (1969). Projection techniques for evaluating surgery in Parkinson's disease. In F.J. Gillingham \& I.M. Donaldson (Eds.), Third symposium on Parkinson's disease (pp. 151-157). Edinburgh, Scotland: Livingstone.

Sharpe, M.H. (1990). Distractibility in early Parkinson's disease. Cortex, 26, 239-246.

Sharpe, M.H. (1992). Auditory attention in early Parkinson's disease: An impairment in focussed attention. Neuropsychologia, 30, 101-106.

Shiffrin, R.M., \& Schneider, W. (1977). Controlled and automatic human information processing. II. Perceptual learning, automatic attending, and a general theory. Psychological Review, 84, 127-190.

Stern, M.B. (1988). The clinical characteristics of Parkinson's disease and parkinsonian syndromes: Diagnosis and assessment. In M.B. Stern \& H.I. Hurting (Eds.), The comprehensive management of Parkinson's disease (pp. 3-50). New York: PMA Publishing Corp.

Stern, Y. (1987). The basal ganglia and intellectual function. In J.S. Schneider \& T.I. Lindsey (Eds.), The basal ganglia and behavior. Toronto: Hans Huber Publishers.

Stern, Y., Mayeux, R., \& Rosen J. (1984). Contribution of perceptual motor dysfunction to construction and tracing disturbances in Parkinson's disease. Journal of Neurology, Neurosurgery, and Psychiatry, 47, 983-989.

Stern, Y., Sano, M., Paulson, J., \& Mayeux, R. (1987). Modified Mini-Mental State Examination: Validity and reliability. Neurology, 37 (Suppl 1), 179.

Stern, Y., Tetrud, J.W., Martin, W.R.W., Kutner, S.J., \& Langston, J.W. (1990). Cognitive changes following MPTP exposure. Neurology, 40, 261-264.

Stroop, J.R. (1935). Studies of interference in serial verbal reactions. Journal of Experimental Psychology, 18, 643-662.

Talland, G.A. (1965). Deranged memory. New York: Academic Press.

Taylor, A.E., Saint-Cyr, J.A., \& Lang, A.E. (1987). Parkinson's disease: Cognitive changes in relation to treatment response. Brain, 110, 35-51.

Wechsler, D. (1945). A standardized memory scale for clinical use. Journal of Psychology, 19, 87-95.

Wechsler, D. (1955). The Wechsler Adult Intelligence Scale. New York: Psychological Corporation.

Weingartner, H., Burns, S., Diebel, R., \& LeWitt, P.A. (1984). Cognitive impairments in Parkinson's disease: Distinguishing between effort-demanding and automatic cognitive processes. Psychiatry Research, 11, 223-235. 